\title{
EEG Emotion Signal of Artificial Neural Network by using Capsule Network
}

\author{
Usman $\mathrm{Ali}^{1}$, Haifang $\mathrm{Li}^{*}, 2$, Rong $\mathrm{Yao}^{3}$, Qianshan Wang ${ }^{4}$, Waqar Hussain ${ }^{5}$ \\ Syed Badar ud Duja ${ }^{6}$, Muhammad Amjad ${ }^{7}$, Bilal Ahmed ${ }^{8}$ \\ College of Information and Compute, Taiyuan University of Technology, Taiyuan, Shanxi, China
}

\begin{abstract}
Human emotion recognition through electroencephalographic (EEG) signals is becoming attractive. Several evolutions used for our research mechanism technology to describe two different primaries: one used for combining the vital attribute, frequency sphere, and physical element of the EEG signals, and the architecture describes the two-dimensional image. Emotion realization is imposing effort in the computer brain interface field, which is mostly used to understand the field of education, medical military, and many others. The allocation issue arises in the required area of emotion recognition. In this paper, the allocation structure based on Caps Net neural network is described. The heder factor shows that the best point to classified the original EEG signals scarce group to using many of the algorithms like Lasso for a better function to used and other than occupy the heights.Furthermore, essential features like tiny subset take by input for the computer network attain for many ultimate emotional classifications. Many of the results show to alternate the best parameters model use and other network formats to making the Caps Net and another neural network act as the emotional valuation on EEG signals. It attains almost $80.22 \%$ and $85.41 \%$ average allocation efficiency under demeanor and view of the emotion pathway as compared to the Support Vector Machine (SVM) and convolutional neural network(CNN or ConvNet). A significant allocation edge attains the best conclusion and automatically enhances the performance of the EEG emotional classification. Deep learning access, such as CNN has widely used to improve primary allocation performance of motor symbolism-based brain-computer interfaces (BCI). As we know that CNN's limited allocation achievement degraded when an essential point data is distorted. Basically, in the electroencephalography (EEG) case, the signals consist of the same user are not measure. So we implement the Capsule networks (CapsNet), which is essential to extract many features. By that, it attains a much more powerful and positive performance than the old CNN approaches.
\end{abstract}

Keywords-Emotion recognition; caps net; EEG signal; multidimensional feature; hybrid neural networks; CNN; Granger; motor imagery classification; deep learning

\section{INTRODUCTION}

Emotion is a sentimental state of human beings and plays a significant role in making decisions and social interactions. Most of the emotional pictures display out the human nerves system and other mental systems to sense and understand human emotion with the help of the computer system, especially in human-computer interaction. Many machines are able to present the human emotion and other nerves terminology. In a present research method, emotion realization is mostly separated by the other two classes[26]. One thing is the critical castigatory depends on the non-physiological signal such that the speech recognition body of the human attitude and other facial expressions. However, other necessary and essential thing is to use physiological signals like myoelectric signal ECG, EEG, and its signal are mostly attaining the cerebral codex's changes directly reflect the human emotion in the current state. The basic research approach of emotion reorganization for the important branches divided into two different classes[14]. Class I is used to show the fundamental category depends on the signal and other nonphysiological states like speech, body attitude, and the other face term. Another necessary and essential thing is used physiological signals like the myoelectric, ECG, EEG beside these EEG signals are directed to attain the deep layer because all the other human emotions will be change by reflecting straight[8].

Fig. 1 shows the architecture of the CapsNet. EEG used for divided into more emotional signals. In this paper, one Caps Net neural network used for priority. Stained flow scheme mostly used for making Caps Net neural network structure to imagine the EEG emotion sensor and comparison to classified by using another relevant algorithm. Now a days, different approaches are used such as the sense-based control system and track approaches to increase the collaboration with other devices. Brain-computer interface (BCI) is the best technology used for defect people[24]. So, this technology is used to provide a comfortable environment to improve the defect person. Brain-computer interface (BCI) tented approaches to deliver the technology to help defect person indirectly by using the mechanism of the machine without any other physical cooperation to transform by the electroencephalogram achievement. The nerves system of the human called neurotransmission movement used for supervising the direct way of the sequential preprocessing. In this paper, we used the eradication and transcription procedure, which is generally used in Brain-computer interface (BCI) in EEG signal and SMR appears whenever motor symbolism and movement[1]. A fundamental method is discuses to apply any Cap-Net to divide two major classes used for the EEG signal for imagery motor [11]. We always have seen that Caps Net escaped about the conflicting signal that used for the symbolism motor, and the other is determined. If we want to make the efficient architecture of the Caps Net to sort the signals. This technique is precious using the primary signals to see the clear image of the EEG that is converted 2D pictures to other STFT algorithms using the necessary convert signals to the domain timefrequency[19].

\footnotetext{
*Corresponding Author
} 


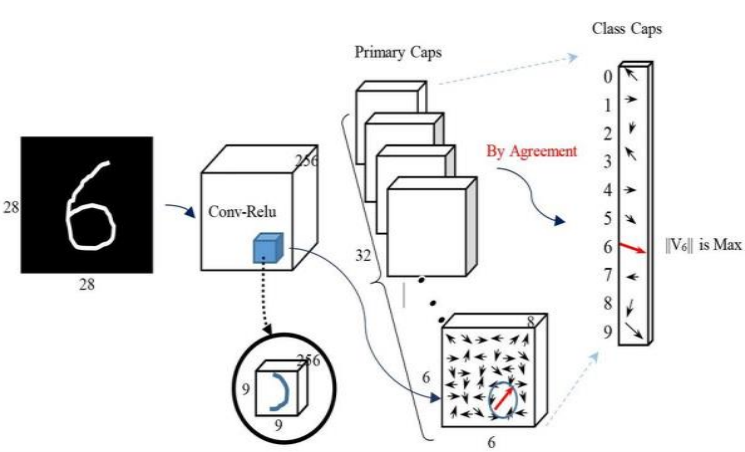

Fig. 1. CapsNet Architecture.

\section{RELATED WORK}

\section{A. EEG Feature Extraction}

This study shows that the broad range of the EEG important aspect of many of the other eradication approaches for prescribe the last 12 years. Many of the essential old EEG features are used for the extraction approaches for the total focus on the frequency dimension and other related dimension information. This time is the sphere for the study about the EEG signal with the proper sequence of series[23]. Timedomain approaches are not used for the prevent. Moreover, much learning has done for human emotion like all the domain time for the better attribute. If you say about the frequency domain, the EEG signal by the relevant area uses the power of the divide-frequency approach like the alpha, beta, theta, and last is delta attribute. A crucial significant algorithm is used Fast Fourier Transform (FFT) Another opportunity we can use as an algorithm called short-time furrier Transform (STFT), and Power Spectral Density (PSD) is widely used for better understanding EEG[3]. Since the EEG signal is non-static for the people to describe the best and too much newly connecting by using the time factor, and another important thing is the frequency for the best domain access the extra information. Hilbert-Huang Transform (HHT) is one of the best learning about the EEG signal and other frequency domain. We always divided into the signal and other Intrinsic Mode Functions (IMF) by using the new trend and other than access rapid frequency data. So, the EEG feature al about the music linking subject for better understanding[18].There are many time frequencies used for the better resistant to over the noise other than the STFT based feature. First is the most frequency feature is used called (HHT) to improving the eradication and many more scale for the collapse as the EEG emotional features. So, if we say all the relevant results to first is to determine the time-frequency function to accept the most relevant result rather than the domain of the old signal for the best feature[1].We always see a better understanding of the most considerable interaction for the spatial like the time and other relevant frequency and different dimensions[9]. Moreover, the old study is all about the paint attraction to the appropriate domain. The relevant information is the best way describe the study were used to the limitation of the inequality used for the different aspect between the electrode combination an essential method is used for the calculated difference in the dominant line of the many other corresponding electrodes combination for the right and left a fraction of the scale [13]. EEG action I always used the sequence of the topologies for preventing many of the other separated multi-spectral picture for the learning about the subjective function. However, sometimes, we can see that the minor emotions used with additional spatial information [10]. An essential component of the EEG, many other directions for viewing the different Constance, established the proper circulation of the EEG electrodes (20-29) scheme of the map better frequency domain the many of the other two dimensions picture. So, a better method is under for the sequence of the image from the successive time for different EEG signals[20]. Fig.2 shows the mechanism of feature extraction used in different neural models. TABLE I shows the EEG emotion extraction of artificial neural network with different methods.

\section{B. Emotional model}

Different models are used for emotion classification, but two significant emotion models are used to understand the EEG signals. First, a necessary and essential method is used to accept a person's emotion design. All emotions are divided into six fundamental thing dislike, hearted, delight, scare, shock, sorrow. The phenomena are elaborate to hold the human emotion is ultimately showing the necessary person's emotion. Besides this, another model is used called a twodimensional emotional model to describe the vision of the emotion of the multi-dimension[27].

The emotional element focuses on the two-dimensional plan. The first emotional step dimension represents the valence that meant by the emotional state of the person to show the range between the negative and positive emotions (Angry, Negative). The second dimension is describing the arousal. It meant that the emotional depth to feel any person. This dimension is also critical because all the simplicity and adaptability describe in this dimension. Valence-Arousal model is a beneficial classification model for better understanding[1]. Fig. 3 represents the different human emotions.

\section{Emotion Classification Methods}

In this paper we provide the comparison of the different methods to elaborate human emotional classification based on valance and Arousal. In below figure show the rating of the accuracy and the many other object counts as a number. In below table describes the most commonly emotional classification approaches included the K-Nearest Neighbor, that is used for the support of the vector machine represented by the SVM that is used mostly. This method is always used for the baseline method and another comparison method for help to understand this model[17].

One of the meaningful method listed to the classified by the motion of the statics and except for a new technique used in accept by learning the LSTM/RNN by using the EEG features increase periodically and dynamic[4]. Many points are used to discuss the EEG model, but there are two different points applied to the best reason for selecting the CNN and LSTM/RNN is the best part for the classification method. Another column of the table is used to show the primary classification of the different numbers of the class used in the previous study. In early research is used for the essentially divide the emotion into a subcategory [33].TABLE II shows a summary of different studies on human emotions using different neural models. 


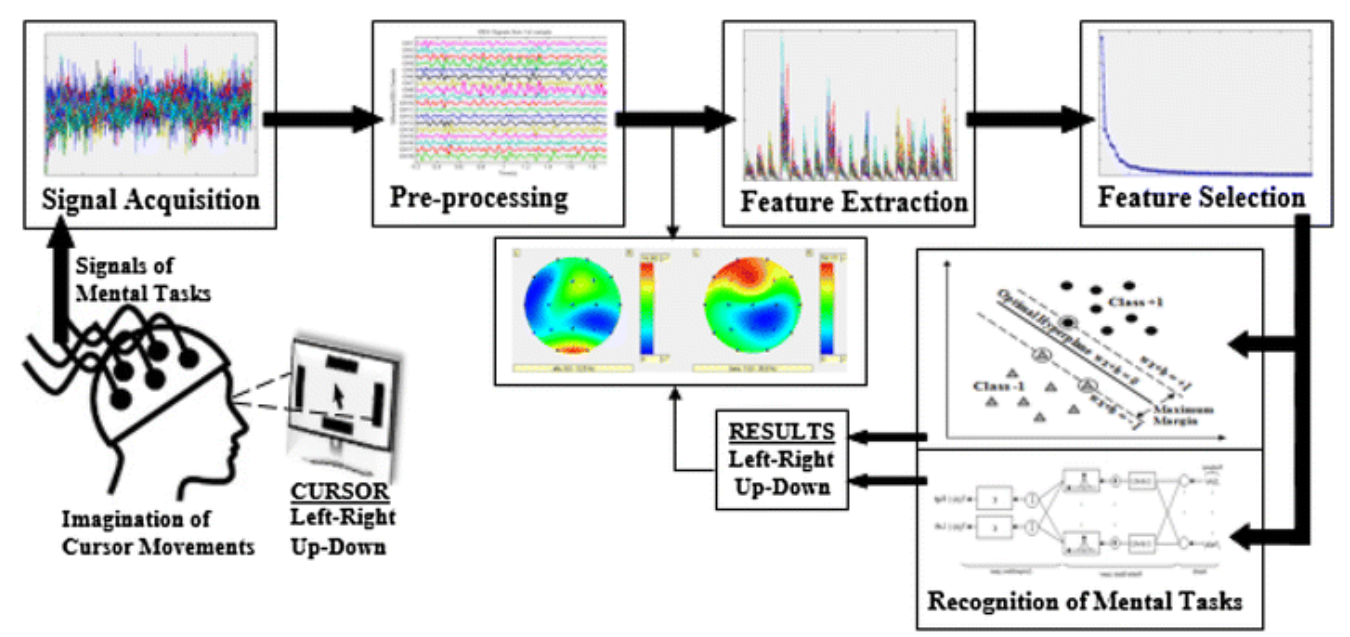

Fig. 2. EEG Feature Extraction.

TABLE. I. EEG SUMMARY FOR EMOTION EXTRACTION

\begin{tabular}{|c|c|c|c|c|}
\hline Author and Study & Year & EEG Feature & Extraction Method & Dimension \\
\hline Agrafioti et al.[1] & 2011 & Activity, Mobility, Complexity & Servick's Method & Time \\
\hline Baird et al.[3] & 2011 & 9 Sub-band of EEG $(6-22 \mathrm{~Hz})$ & STFT & Frequency \\
\hline Candra et al.[6] & 2015 & Activity, Mobility, Complexity & Welch's Method & Time \\
\hline Daugbjerg et al.[8] & 2018 & Sub-band:0, $\alpha, \beta, \gamma$ & FFT & Frequency \\
\hline Mislove et al.[23] & 2007 & EEG $\gamma$ band $(32-100 \mathrm{~Hz})$ & FFT & Frequency \\
\hline Orlowski et al.[25] & 2010 & Higher order crossing & DWT & Time \\
\hline Xin et al.[16] & 2017 & $\begin{array}{l}\text { Sub-band } \delta, 0, \alpha(9-14 \mathrm{~Hz}), \beta(2-29 \mathrm{~Hz}), \gamma(36- \\
40 \mathrm{~Hz})\end{array}$ & STFT & Frequency \\
\hline Thammasan et al.[29] & 2016 & Sub-band:0, $\alpha, \beta, \gamma$, NLD, NSI & Welch's Method & Frequency \\
\hline Lua et al.[22] & 2011 & $\beta / \alpha$ Sub-band: $\beta$ & FFT & Frequency \\
\hline Kim et al.[18] & 2007 & HHS based feature vectors & HHS & Time and Frequency \\
\hline Ghassemlooy et al.[15] & 2019 & Change and asymmetry in sub-band of $\alpha$ & Welch's Method & Frequency and Special \\
\hline Crocioni et al.[7] & 2007 & Spectral power and difference & FFT & Frequency and Special \\
\hline Bonheur et al.[5] & 2019 & $\begin{array}{l}\text { Correlation, Coherence and phase } \\
\text { synchronization }\end{array}$ & FFT & Frequency \\
\hline Easley et al.[11] & 2018 & PSD, DE, DASM and RASM & STFT & Frequency \\
\hline Lee et al.[19] & 2014 & Density Estimate & $\begin{array}{l}\text { Kernel Estimation } \\
\text { Density }\end{array}$ & Frequency \\
\hline Murugappan et al.[24] & 2010 & Sub-band: $0, \alpha, \beta$, & MDFDA & Frequency \\
\hline Yan et al[.[32] & 2014 & $\begin{array}{l}\text { Sum of Squared of absolute values of sub-band: } \\
0, \alpha, \beta\end{array}$ & FFT & Frequency and Special \\
\hline Skarsgard et al.[28] & 2008 & $\begin{array}{l}\text { Fractal Dimension (FD) and Power Spectral } \\
\text { Density (PSD) }\end{array}$ & Welch's Method & Frequency \\
\hline Petrantonakis et al.[27] & 2010 & PSD, DE, DASM and RASM, ASM and DCAU & STFT & Frequency \\
\hline Hunt et al.[16] & 2012 & Multi-scale Entropy & HHT & Time and Frequency \\
\hline Frieden et al.[14] & 2006 & Frequency Features and Time-Frequency Feature & FFT & Time and Frequency \\
\hline
\end{tabular}




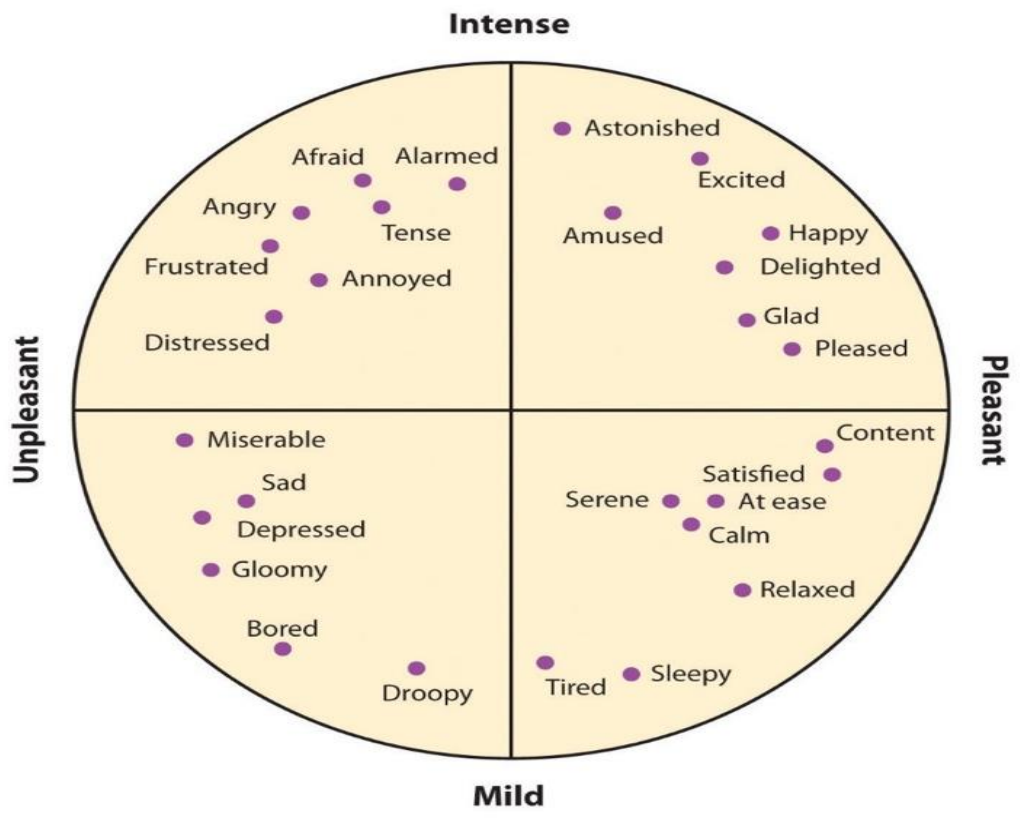

Fig. 3. Different Human Emotion.

TABLE. II. EEG SUMMARY FOR EMOTION EXTRACTION

\begin{tabular}{|l|l|l|l|l|}
\hline Author and Study & Emotion Classification & Subject & Accuracy & Classification Method \\
\hline Baccelli[2] & Valence and Arousal (2 class) & 10 & $81 \%$ & SVM \\
\hline Fischer[12] & Valence and Arousal (3 class) & 30 & $66.7 \%$ & SVM \\
\hline Jung[17] & Valence and Arousal (2 class, Respectively) & 28 & $81.3 \%$ & SVM \\
\hline Lua[21] & Valence (2 class) & 12 & $71.3 \%$ & K-NN \\
\hline Voellmy[30] & Valence (2 class) & 9 & $82 \%$ & SVM, K-NN \\
\hline Daugbjerg[17] & Valence and Arousal(2 class) & 15 & $82 \%$ & SVM \\
\hline Ghassemlooy[15] & Valence and Arousal (2/3 class, Respectively) & 32 & $66.6 \%, 66.4 \%(2)$ & Bayes Neural Network \\
\hline Oh[25] & Valence and Arousal (2 class, Respectively) & 32 & $74.12 \%$ & C-RNN \\
\hline Voellmy[30] & Valence and Arousal(4 class) & 32 & $75.21 \%$ & CLRNN \\
\hline
\end{tabular}

\section{EEG MFI Progressions}

The universal scheme recognizes the 12-22 is ides for the best design of identity apply for the necessary edge of an amount to electrodes the situation for the EEG testing. This scheme is based on the communication between the best edge of the wires and the other underlying area for the intelligence. The difference shown between the (13 to 23) assigns the essential fact to calculate the real distance between the adjacent electrodes are either the $12 \%$ t or $22 \%$ for the all front-back to the left-right distance of the scalp[22]. An international plan in view roundabout (10-20) system and another generalized squire of the matrix used for it. In the EEG electrodes, point to describe the best test used for the DEAP datasets. We can see that the DEAP dataset is used in the square matrix $(\mathrm{N} \times \mathrm{N})$ [4]. In the following equation, $\mathrm{N}$, we used for the extreme point of the number exist between the vertical and horizontal. In looking for the DEAP dataset, Where $\mathrm{N}$ is equal to the 10 . So, the basic matrixes are used to fill the EEG frequency that represents a side ofthe right of the picture that shows clearly. Thus, the line is gray looks like the triangle that is mention before the midpoint of the square matrixes describes the mansion. Many other color combinations show mostly red point shows the electrodes comparable to the red circle used for the universal (12-22) edge, and the gray color point is inserted of other the full matrixes. The essential cost of red color is showing comparable frequency-feature (PSD) that is used for the EEG electrodes. If we see in the pointed gray that is an addition to the red point neighboring that [31]. Fig 4 represents the EEG MFI node construction. 

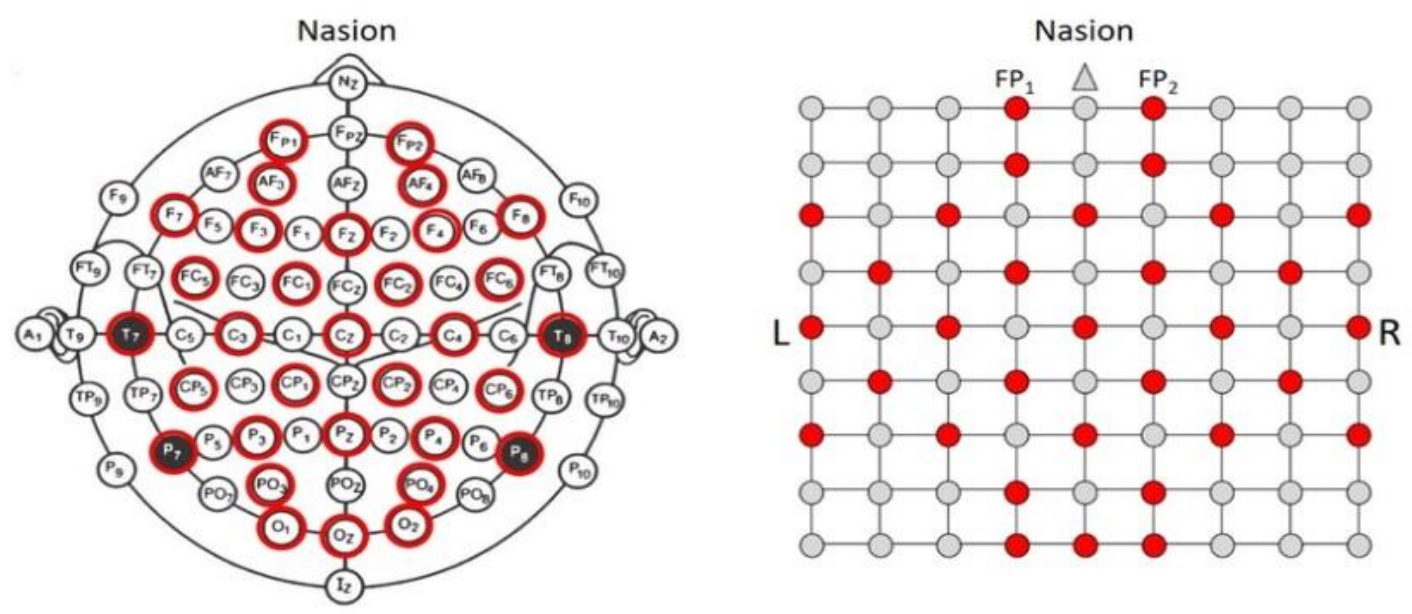

Fig. 4. Sequences of EEG MFI Construction.

\section{CAPS-NET NEURAL NETWORK}

CapsNet neural network is used for the neural vector other than a neural point for the old neural network track of network model changing routing techniques. It is mostly studying the feature of the training data fully classify the details[2]. The old CNN neural network is mainly used local classified feature for the speech, video and image fields, moreover CNN demands to complete a copy of the feature detector. If we draw a design for the spatial information that can diminish the efficiency of one structure. There is not a limited efficiency model. Still, sometimes linear and distinct data not use for the EEG signals,
CNN neural network not adequately described for the EEG signal features and other classify efficiency changing routing technique in Caps Net network that can create a fact between lowest level and heights level point. Which can be used as an intelligent model for the better relationship between the local and all other models. That is automatically applying the network to sense for the new climate[25]. Fig5 represents the CapsNet neural network model related to human emotion signals.

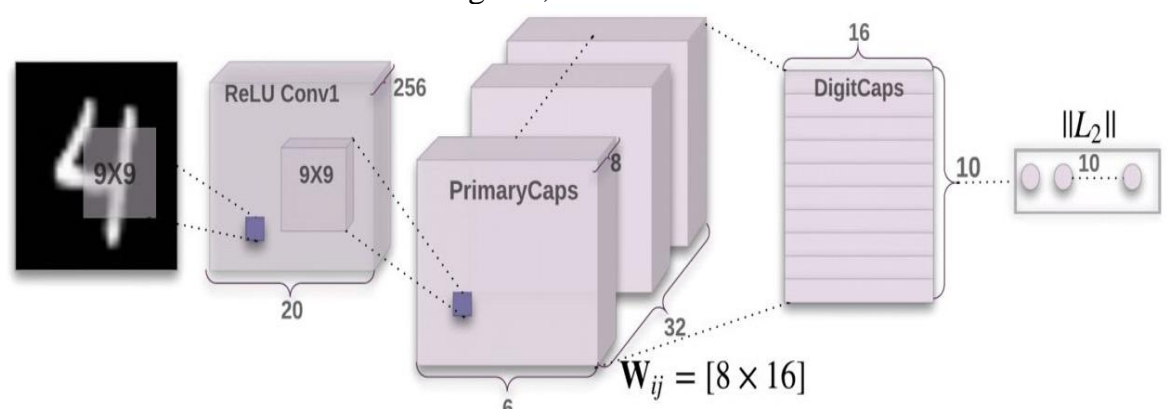

Fig. 5. CapsNet Neural Network.

\section{DEEP LEARNING BASED IN CNN}

Deep learning based on the access for the two different picture qualification techniques, first is the CNN and another is the Cap-Net. Both are used for the general classification task. We can concert the AD EEG signals into the 2D picture by using the STFT algorithm. STFT algorithm produces the 2D time-frequency sphere picture from any channel like electrodes [25]. For example, if we want to use the EEG signal from the 3 or 4 electrodes, then 3 channels are using the 2D image data look like RGB picture generating and use the testing and training of the classifier. When we calculated the suppress function that can act like the non-linear active function of $\mathrm{CNN}$ to develop and used the training and the testing of the classifier. Many of the other details about the generation in 2D STFT picture is used for the calculated 3 electrodes with the examiner rate is $255 \mathrm{~Hz}$ is described before. After using the EEG signal to accept the notch-filtered that is used for the separation according to the best action performs before. We can use the data of the array size $4 \times 2 \times$ 400. If we apply the algorithm STFT, we can draw the $2 \mathrm{D}$ data array size $79 \times 16$ which is used for the X-axis and $y$-axis that signed to the frequency and time respectively[6].In EEG, signals can be divided into different band frequency like Alpha $(9-18 \mathrm{~Hz})$, Beta $(18-33 \mathrm{~Hz})$, Gamma $(>34 \mathrm{~Hz})$, Theta $(5-$ $8 \mathrm{~Hz})$, Delta $(<5 \mathrm{~Hz})$, and $\mathrm{Mu}(9-13 \mathrm{~Hz})$. They all depend on the desire and operation of the study for the specific range that can be calculated by the signals (e.g., mu and other Beta Bands) which is selective. For motor symbolism allocation tasks like the frequency band, $6-32 \mathrm{~Hz}$ are commonly used. In this paper, we study to choose the frequency range calculates the $9-31 \mathrm{~Hz}$ for the quotation of the STFT spectrogram picture. So, in the end, we can see 3 channel 14 x 15 image in capsule networks that can be used as a testing and training thoroughly[7]. Clarify the network design to propose the Caps Net-based path. At the same original Caps-Net paper takes out 
the $2 \mathrm{D}$ picture as the starting input. We can see in our architecture layer (kernel size: 8x8 with 2 strides 2), another dropout layer before we can use the Caps layer, the result shows the 18 dimensions as per class (Left-Hand as compare to the Right-Hand). The training of the Caps Net is also complete. The versatile routing by agreement exists between the capsule as detailed before. The learning ability that can be feasible by the Caps Net on the EEG domain also explained in the previous section[30].Fig 6 shows the deep learning CNN model.

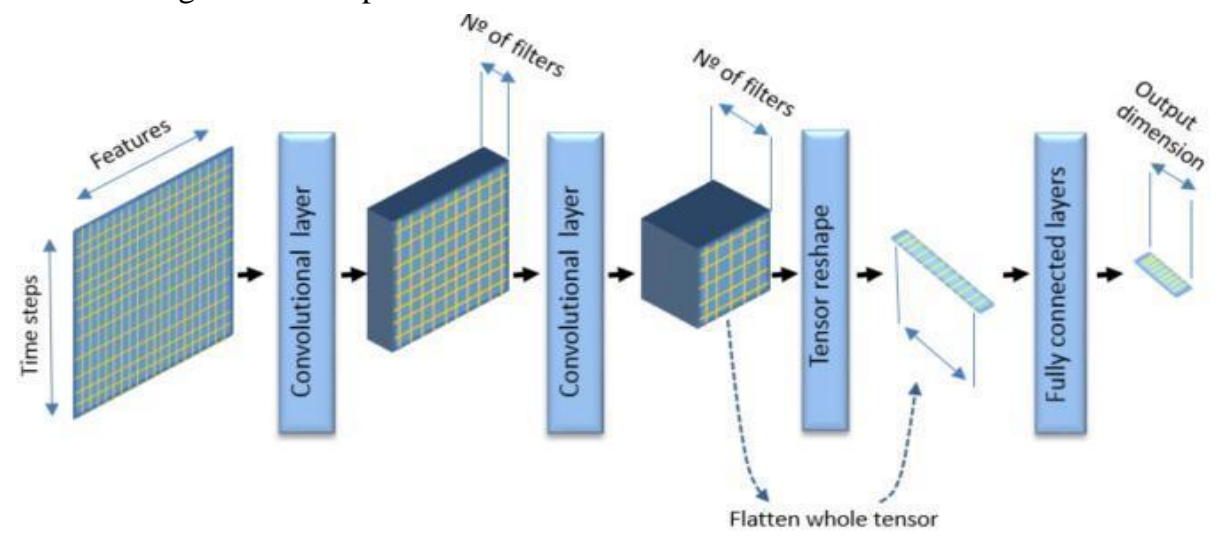

Fig. 6. Deep Learning in CNN.

\section{CONVOLUTIONAL NEURAL NETWORKS}

The necessary input used for MFI is to calculate the size $250 \times 198$ pixels, and it consists of three different colors of channel. Many of the set numbers that can be used for the twist filtering as 28 for the first convolutional layer to be use the excerpt for the 29 different types of correlation information name as the 28 various features, In that time we extract the different ratio dimensional layer[28]. There are many sizes of the field used like $5 \times 6$ pixels and $8 \times 8$ pixels commonly. Usually there are different sizes used like the stalk 3,6 and 11 pixels because it won't have any overlap and strides. The primary activation action is used like ReLU[7]. The firstly ordinary layers used in max-pooling and this pooling size is $3 \times 3$, these are the strides. A second regular layer uses 10 different filtering with their size 4 x 4 without any overlap between strides. Further integrate information is always helpful for the exclusive range for the previous feature's unit is used for connecting. A smash operation has occupied the change of the final feature into the different spatial functions of the vector. The basic structure of $\mathrm{CNN}$ is explained earlier. The solid layer changes the final feature into the one-spatial feature vector. Looking is this layer; we can easily set the output $1 / 11$ of the soloed layer. Furthermore RNN output layer SoftMax is used as the real activate function for the exact output size prior to other 5 corresponding emotion state[21].

Table III shows the specifications of different CNN layers.

TABLE. III. CONVOLUTIONAL NeURAL Networks

\begin{tabular}{|c|c|}
\hline Layers & Specification \\
\hline Convolution (Conv) 1a & 64 (Filter: $3 \times 3 \times 3$ and stride: $1 \times 1 \times 1$ ) \\
\hline Pooling 1 & $1 \times 2 \times 1$ \\
\hline Conv.2a & 128(Filter: $3 \times 3 \times 3$ and stride: $1 \times 1 \times 1$ ) \\
\hline Pooling 2 & $2 \times 2 \times 2$ \\
\hline Conv.3a & 256(Filter: $3 \times 3 \times 3$ and stride: $1 \times 1 \times 1$ ) \\
\hline Conv. $3 b$ & 256(Filter: $3 \times 3 \times 3$ and stride: $1 \times 1 \times 1$ ) \\
\hline Pooling 3 & $2 \times 2 \times 2$ \\
\hline Conv.4a & 512(Filter: $3 \times 3 \times 3$ and stride: $1 \times 1 \times 1$ ) \\
\hline Conv.4b & 512(Filter: $3 \times 3 \times 3$ and stride: $1 \times 1 \times 1$ ) \\
\hline Conv.5b & 512(Filter: $3 \times 3 \times 3$ and stride: $1 \times 1 \times 1$ ) \\
\hline Pooling 5 & $2 \times 2 \times 2$ \\
\hline Fully Connected (FC) & 4096 \\
\hline FC & 4096 \\
\hline Soft Max & Output \\
\hline
\end{tabular}




\section{LSTM RECURRENT NEURAL NETWORK}

Many of the in-depth analysis of simulation emotion change up the 70s. This emotion is calculating by the different subjects often fare the important moving part to all videos. Moreover, we want to describe a structure for the text information for many long distances as per sequence. In the previous look the RNN is the better sequential structure[29]. Simple RNN challenges see or in gradient vanish, or others see in the collapse in the back reproduction. So, the LSTM unit is used for the accept the easy group for old RNN, and the LSTM unit is the combination of the entering mechanism for the better structure[12]. So, data timing is most useful thing to manage and carry easily for the long during for best computation, the doorway is used for the necessary instruction and another individual -loop mechanism. That allows the gradient to move very long durations[30].A standard design that is used in the LSTM unit to describe in the below figure. If we compare two different structures of the neural network units. There are two units illustrated in the below picture and comparison between the recurrent neural network and LSTM [32]. In the picture, we can see that RNN is used for the content of the evolution of the output that given input. The other LSTM is used for the content the three basic gate structure like (input gate, forget gate, and output gate) that is used to observe what kind of the information is move from the previous step and it should be erased the information at the current time for added the main data flow [16].Fig 7 shows the functioning of LSTM Recurrent Neural Network.

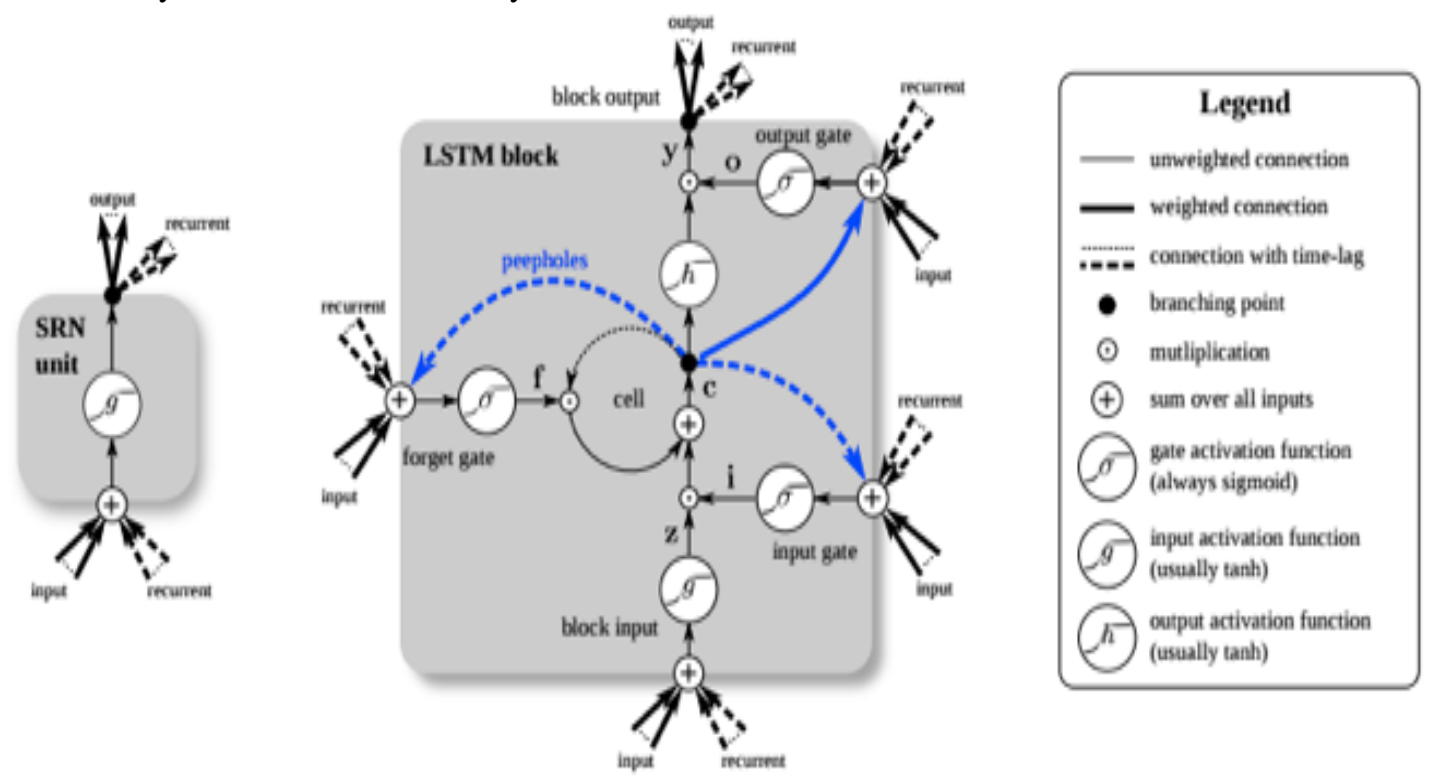

Fig. 7. LSTM Recurrent Neural Network.

\section{RESULTS AND DISCUSSION}

\section{A. Dataset and Preprocessing}

We can calculate the actual work expected into the Caps Net using basic on the best path on the BCI dataset. We can organize the massive experiment by using the BCI competition VI 2c datasets. That dataset can be a subset of EEG signals which can calculate the essential 10 subjects between two different class motor symbolism function. For example (left side and right side).There are 4 primary bipolar channels used like (C2, C5, and C6) for the EEG that can be recorded with different frequency of $260 \mathrm{~Hz}$. Also, there are many signals existing between 0.8 and $123 \mathrm{~Hz}$ used for the filter bandpass bit notch filter also apply for $54 \mathrm{~Hz}$ frequency can handle almost 6 data set consist in their session. The first four sessions are used for the practice, and other is used for the testing. The average of the training data for each subject is 450 trails to count $(200+200+50)$ where test data just for the $300(150+150)$. By using the experiment to count the effectiveness of the dataset. A classifier is used for the train and test subject by subject experiment can be done according to the guidelines of the data set for the relevant classifier is used for the test and training motor-imagery activation. There are many semi motors to capture the $\mu$ and $\beta$ bands. Furthermore, an excellent frequency band is used. For example $(9 \mathrm{~Hz} \sim 90 \mathrm{~Hz})$ are mostly chosen by the feature of extraction. A good understanding of the EEG division used between the 0.7 to 3.1 that can be greeted by a better classification result. There are many experiments used for the EEG original signals from the dataset that have filtered the band pass exist between the $9 \sim 33 \mathrm{~Hz}$ and just 3 EEG segment $(0.7 \sim 3.5 \mathrm{~s})$ is used for processing the features. A significant network can be used to analyze the complete Brain Decode framework. A lot of features can be used for the Processing of EEG. The study can be controlled by the desktop PC computers will be used called X GPU. Fig 8 shows different datasets of CapsNet. 


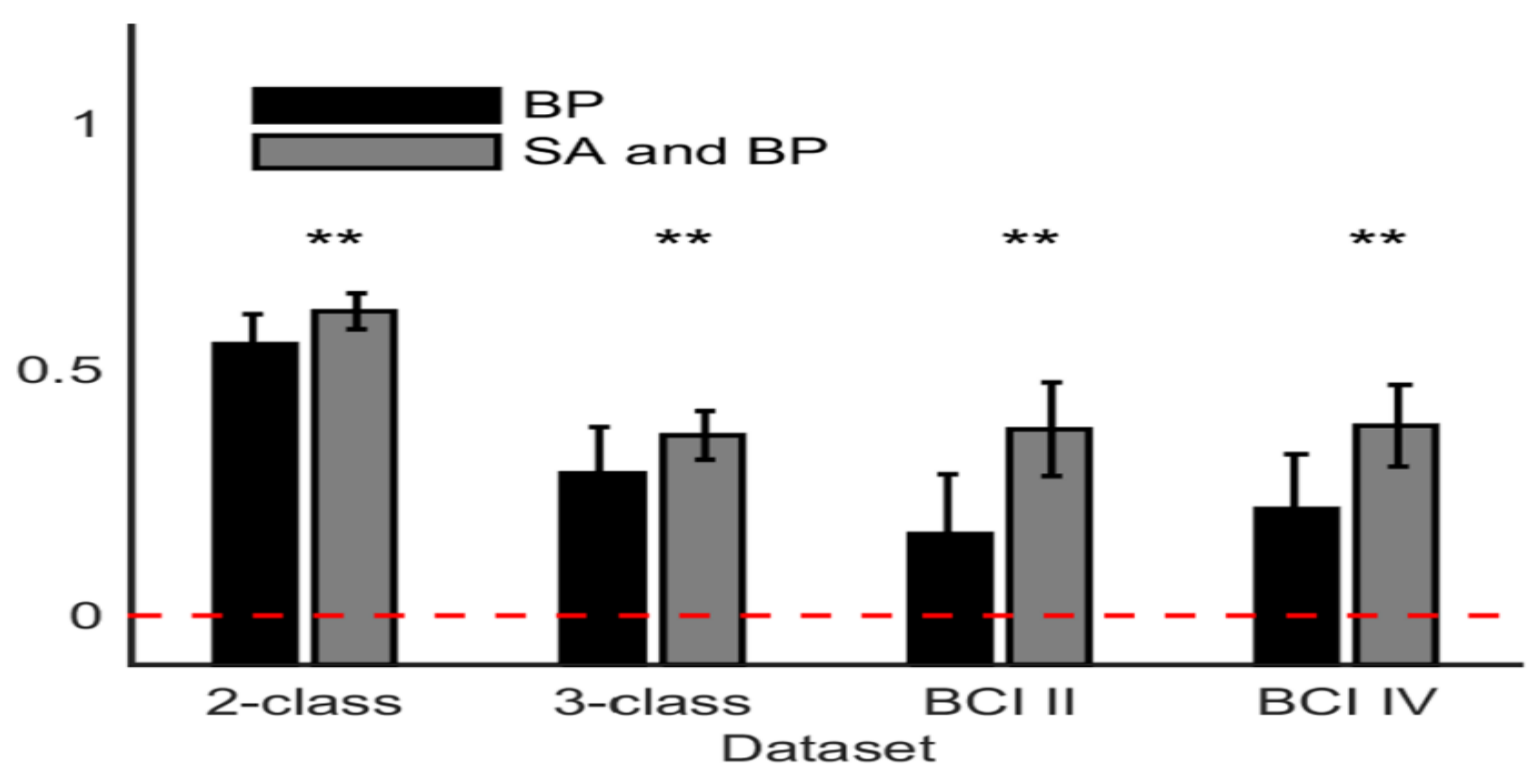

Fig. 8. BCI Dataset of CapsNet.

\section{B. Performance Evaluation}

Firstly, we can calculate the feasibility for apply the Caps Net on the EEG motor symbolism for the different analysis that can be used for the original design to better picture and point classification. The Caps Net is trained for every case with the 35 initial capsule that is the best pathway used 9 and 19 detention for MI caps with 2 output right and left. The Caps Net is trained for the best EEG sphere with the STFT picture. We can see that the test and train are decreased fast within 15 times and gradually concentrate for 3 times. The best performance is used many terms to reduce the best efficiency express 3 minutes classification rates like the many numbers of the trails out of all paths. The Caps Net attain $86 \%$ of different classification efficiency. When we use many numbers of routing repetition is set to 6 . It does not found any vital effects that can be used for the number of repetitions to classify the accurate network. We can check the initial results of the process after repair for best performance. Including the best analysis, the learning ability for the Caps Net for the EEG signal domain. If we can make a comparison between the different classification of the accuracy used for the expected Caps Net based approach with the old CNN based approach, in comparison of different Caps Net apply for the best suggestion by the 2 different involutions and pooling layer for the different decryption motor for the symbolism signal using SFTF picture. If we test and train, the input of the CNN approaches in the same STFT picture using the 6 channels $16 \times 162 \mathrm{D}$ vectors. In this analysis, the primary classification can be accurate and best average calculation scores for the 15 different subjects evaluates. TABLE 4 shows the comparison between CNN and CapsNet. Fig. 9 shows graphic view of this comparison.

TABLE. IV. COMPARISON BETWEEN THE CNN AND CAPSNET

\begin{tabular}{|l|l|l|}
\hline Subject & CNN & CapsNet (Planned) \\
\hline 01 & $71.72 \%$ & $77.01 \%$ \\
\hline 02 & $51.15 \%$ & $59.54 \%$ \\
\hline 03 & $54.18 \%$ & $49.12 \%$ \\
\hline 04 & $90.38 \%$ & $91.23 \%$ \\
\hline 05 & $80.18 \%$ & $84.25 \%$ \\
\hline 06 & $74.01 \%$ & $92.19 \%$ \\
\hline 07 & $71.25 \%$ & $71.24 \%$ \\
\hline 08 & $84.58 \%$ & $88.21 \%$ \\
\hline 09 & $83.41 \%$ & $80.31 \%$ \\
\hline Average & $73.43 \%$ & $70.01 \%$ \\
\hline
\end{tabular}




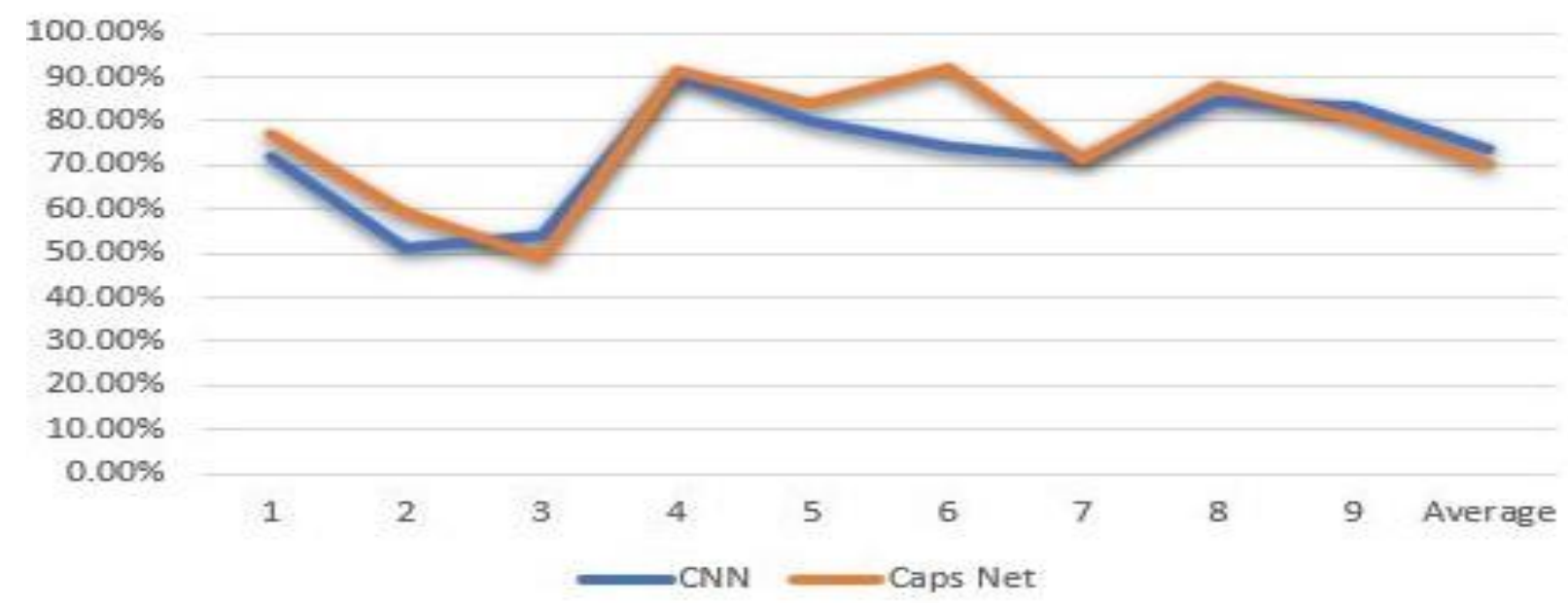

Fig. 9. Comparison between the $\mathrm{CNN}$ and CapsNet.

\section{CONCLUSIONS}

In this paper, firstly classify the Caps-Net neural network and observe the primary emotion of the EEG signals. The total result must be shown that the Caps-Net achieves precise information collection rather than the old neural network. There are many useful emotional classifications of the EEG signal. At the same time, the basic design and framework are adopted to the Caps Net. Calculate the better performance and feasibility that will describe the different methods for many experiments on BCI completion was attended. Another experimental result conducted previously for different methods of using CNN based two class motor imagery tests. Moreover, the improvement of the Caps-Net based approach uses EEG decryption frequency features (PSD) are used to exact the EEG channel and made the two-dimensional design for construct the EEG MFI. So, the necessary signals of EEG MI are building for the apparent sequence of the raw signals. Hybrid deep neural networks are connected to the primary convolution of the Neural Network, and many of the belowterm memory recurrent neural networking hybrid design $\mathrm{CNN}$ is used for the sense of temporary picture EEG MFI sequence of the pattern. To clarify, the LSM/RNN is used for human emotions. If we look at the future side, there are many deep searches employed for classify the underlying protocol of the EEG signal.

\section{REFERENCES}

[1] F.Agrafioti, D. Hatzinakos, and A.K. Anderson "ECG pattern analysis for emotion detection", IEEE T AFFECT COMPUT., vol. 3, no.1, pp. 102-115, 2011.

[2] F. Baccelli and B. Błaszczyszyn, "Stochastic geometry and wireless networks: Volume II Applications," Foundations and Trends® ${ }^{\circledR}$ in Networking., vol. 4, no.1-2, pp. 1-312, 2010.

[3] R. Baird, G. Eeson, A.Safavi, P. Puligandla, J. M. Laberge, E. D.Skarsgard and C. P. S. Netorks, "Institutional practice and outcome variation in the management of congenital diaphragmatic hernia and gastroschisis in Canada: a report from the Canadian Pediatric Surgery Network," J. Pediatr. Surg., vol. 46, no.5, pp. 801-807, 2011.

[4] L. Bilge, T. Strufe, D. Balzarotti, and E. Kirda, "All your contacts are belong to us: automated identity theft attacks on social networks, ". Proc. 18th Int. Conf. W.W.W, ACM., 2009.

[5] S.Bonheur, D. Štern,C. Payer, M. Pienn, H. Olschewski and M. Urschler, "Matwo-CapsNet: "A Multi-label Semantic Segmentation
Capsules Network," Int. Conf. Med. Image. Comput. Comput. Assist. Interv., Springer,2019.

[6] H. Candra, M. Yuwono, R.Chai, A, Handojoseno, I. Elamvazuthi, H.T. Nguyen and S.Su, "Investigation of window size in classification of EEG-emotion signal with wavelet entropy and support vector machine," 2015 37thAnnual int. conf.IEEE Eng. Med. Biol. Soc., pp.7250-7253 .

[7] P. Crocioni, "Net neutrality in Europe: Desperately seeking a market failure," Telecommun. Policy., vol. 35, no. 1, pp.1-11,2011.

[8] C. Daugbjerg, "Policy networks under pressure: pollution control, policy reform and the power of farmers, Routledge," 2018.

[9] A. Dhamdhere and C. Dovrolis, "Can ISPs be profitable without violating "network neutralit"?," Proceedings of the 3rd international workshop on Economics of networked systems, ACM.,pp.13-18,2008.

[10] M. Dischinger, A. Haeberlen, K.P. Gummadi and S. Saroiu, "Characterizing residential broadband networks," (2007).. Internet measurement conference, vol. 7, pp. 43-56.

[11] R. Easley and F. H. Guo, "Research Commentary—From Net Neutrality to Data Neutrality: A Techno-Economic Framework and Research Agenda." Info. Syst. Res., vol. 29, no.2, pp. 253-272, 2018.

[12] A.Fischer, J. F. Botero, M.T. Beck, H. De Meer and X. Hesselbach, "Virtual network embedding A survey," IEEE Commun. Surv. Tut., vol. 15, no. 4, pp.1888-1906, 2013.

[13] T.Fischer, and C. Krauss, "Deep learning with long short-term memory networks for financial market predictions," Eur. J. Oper. Res., vol. 270, no.2, pp. 654-669, 2018.

[14] R.Frieden, "Network Neutrality or Bias-Handicapping the Odds for a Tiered and Branded Internet," Hastings Comm. \& Ent. LJ ., vol. 29, pp.171,2006.

[15] Z. Ghassemlooy, W. Popoola and S. Rajbhandari, "Optical wireless communications: system and channel modelling with Matlab®, CRC press," 2019.

[16] K.J. Hunt, G. R. Irwin and K.Warwick,"Neural network engineering in dynamic control systems," Springer Science \& Business Media, 2012.

[17] C. Y.Jung, H. Y. Hwang, D. K. Sung and G. U. Hwang, "Enhanced Markov chain model and throughput analysis of the slotted CSMA/CA for IEEE 802.15. 4 under unsaturated traffic conditions." IEEE Transactions on Vehicular Technology, vol.58, no. 1, pp. 473-478, 2008.

[18] S.Kim, S. Pakzad, D.Culler, J. Demmel, G. Fenves, S. Glaser, and M. Turon,"Health monitoring of civil infrastructures using wireless sensor networks," Proceedings of the 6th international conference on Information processing in sensor networks, ACM, pp. 254-263, 2007.

[19] Y.Y. Lee and S. Hsieh, "Classifying different emotional states by means of EEG-based functional connectivity patterns," PloS one, vol.9, no. 4, p.e95415, 2014. 
[20] Y.Li, W. Zheng, Z. Cui, Y. Zong and S. Ge, "EEG emotion recognition based on graph regularized sparse linear regression." Neural Process Lett., vol. 49, no. 2, pp. 555-571, 2019.

[21] R.Luaand K. C. Yow, "Mitigating ddos attacks with transparent and intelligent fast-flux swarm network," IEEE Network, vol. 25, no.4, pp.28-33, 2011.

[22] A.Mert, and A. Akan, "Emotion recognition from EEG signals by using multivariate empirical mode decomposition," Pattern Anal Appl., vol. 21, no.1, pp.81-89, 2018.

[23] A.Mislove, M. Marcon, K. P.Gummadi, P.Druschel and B.Bhattacharjee,"Measurement and analysis of online social networks," Proceedings of the 7th ACM SIGCOMM conference on Internet measurement, ACM, pp. 29-42, 2007.

[24] H.Oh, , G. Labianca and G. H. Chung, "A multilevel model of group social capital," Academy of management review , vol. 31, no.3, pp. 569$582,2006$.

[25] S.Orlowski and R. Wessäly, "SNDlib 1.0 - Survivable network design library," Networks: An International Journal, vol. 55, no.3, pp.276-286, 2010.

[26] P. C.Petrantonakis, and L. J. Hadjileontiadis "Emotion recognition from brain signals using hybrid adaptive filtering and higher order crossings analysis," IEEE Transactions on affective computing, vol. 1, no. 2, pp. 81-97, 2010.
[27] D.Skarsgard, J. Claydon, S. Bouchard, P. C. Kim, S. K. Lee, J. M. Laberge, D. McMillan, P. von. Dadelszen and N. Yanchar,"Canadian Pediatric Surgical Network: a population-based pediatric surgery network and database for analyzing surgical birth defects. The first 100 cases of gastroschisis," J. ped. surg. vol. 43, no. 1, pp. 30-34, 2008.

[28] N.Thammasan, K. Moriyama, K. I. Fukui and M. Numao "Continuous music-emotion recognition based on electroencephalogram." IEICE TRANSACTIONS on Information and Systems, vol. 99, no.4, pp.12341241, 2016.

[29] A.Voellmy and H. Kim, et al. Procera: a language for high-level reactive network control. Proceedings of the first workshop on Hot topics in software defined networks, ACM, 2012.

[30] L.Xin, Q. Xiaoying, S. Xiaoqi, X. Jiali, F. Mengdi and K. Jiannan,"An improved multi-scale entropy algorithm in emotion EEG features extraction." J. Med. Imag Heal Info. vol. 7, no. 2, pp.436-439, 2017.

[31] J.Yan and W. Zheng, "Integrating facial expression and body gesture in videos for emotion recognition," IEICE TRANSACTIONS on Information and Systems, vol. 97, no. pp. 610-613, 2014.

[32] X.Yu and Q. Gu, Citation prediction in heterogeneous bibliographic networks. Proceedings of the 2012 SIAM International Conference on Data Mining, SIAM, 2012.

[33] W.L.Zheng and B.-N. Dong, Multimodal emotion recognition using EEG and eye tracking data. 2014 36th Annual International Conference of the IEEE Engineering in Medicine and Biology Society, IEEE, 2014. 